\title{
miR-126 targeting COLPH3 inhibits the epithelial-mesenchymal transition of gastric cancer BGC-823 cells and reduces cell invasion
}

\author{
Jiancai Ouyang, Fuhu Song, He Li, Rui Yang, Haicheng Huang \\ Department of Anesthesiology, the Third Affiliated Hospital of Southern Medical University, Guangzhou, China
}

\begin{abstract}
The incidence and mortality of gastric cancer have been increasing in recent years. miR-126 and target genes have been studied in gastric cancer, but their studies with Golgi phosphoprotein 3 (GOLPH3) and related pathways in gastric cancer are rarely reported. In the present study, we aimed to investigate the interaction between miR-126 and GOLPH3in the progression of gastric cancer. In this study, we revealed the role of the miR-126GOLPH3 axis in regulating the progression of epithelial-mesenchymal transition (EMT) in BGC-823 cell model. Firstly, tumor tissues and adjacent normal tissues were collected from 45 patients with gastric cancer. We found that the expression of miR-126 in human tumor tissue was significantly lower than in normal tissue using reverse transcription-polymerase chain reaction (RT-PCR). But the GOLPH3 expression was opposite by the detection of immunohistochemistry, RT-PCR and Western blot. Moreover, we predicted miR-126 targeting GOLPH3 by bioinformatics and confirmed the interaction using luciferase reporter gene system; miR-126 inhibited the proliferation, invasion and EMT progression in BGC-823 cells through overexpressing miR-126; miR-126 negative regulated GOLPH3 expression by overexpressing and interfering miR-126. Finally, we found that GOLPH3 could promote proliferation using MTT assay, invasion using Transwell, and EMT progression by inhibiting the expression of E-cadherin, inducing vimentin and N-cadherin in BGC-823 cells. Our results demonstrated that miR-126 inhibits proliferative and invasive ability as well as EMT progression by targeting GOLPH3. This study may provide a new field of vision for targeted treatment of gastric cancer.
\end{abstract}

Key words: Gastric cancer cells; microRNA-126; GOLPH3; invasion; EMT.

Correspondence: Department of Anesthesiology, the Third Affiliated Hospital of Southern Medical University, No. 183, Zhongshan Avenue West, Tianhe District, Guangzhou, Guangdong, China.

E-mail: haicheng_huang@163.com

Contributions: HH, conceived the study and designed the experiments; $\mathrm{HH}$, JO, wrote the manuscript; JO, FS, $\mathrm{HL}$, completed the experiment; JO, RY, analyzed the data and revised the manuscript.

Conflict of interest: The authors declare that they have no competing interests.

Funding: This work was supported by grants from the Young Scientists Fund of the National Natural Science Foundation of China (Grant No. 81601095).

Availability of data and materials: The datasets used and/or analyzed during the current study are available from the corresponding author on reasonable request.

Ethical approval: Ethical approval was obtained for all experimental procedures by the Ethical Committee of the Southern Medical University, Guangzhou, Guangdong, China.

Patient consent for publication: Verbal informed consent was obtained from the patients for their anonymized information to be published in this article. 


\section{Introduction}

Gastric cancer is a common malignant tumor of the digestive system with a very high incidence worldwide, which significantly affects people's quality of life. ${ }^{1}$ The major cause of deaths from gastric cancer is tumor metastasis. ${ }^{2}$ Tumor metastasis is strictly regulated by a variety of genes. These genes play significant roles in promoting or inhibiting tumor metastasis. ${ }^{3}$ Reconstructing or downregulating the expression of oncogenes may be an effective method of treating cancers. ${ }^{4}$ Human tissues express miRNA, which is related to the normal physiological functions and growth of the human body. miRNA regulates the processes of cell growth, differentiation and metabolism. ${ }^{5}$ Golgi phosphoprotein 3 (GOLPH3) is located on the 5 p13 amplification target of human chromosomal genes, where many solid tumors are amplified. ${ }^{6}$ Mammalian rapamycin target protein (mTOR), a factor of the classical pathway PI3K/AKT/mTOR, is negatively regulated by miRNAs to attenuate the malignant progression of cancer. ${ }^{7,8}$ Human miRNAs play a vital role in the occurrence and development of tumors. Among these miRNAs, low miR-126 expression was observed in gastric carcinoma and the expression of miR-126 was correlated with the survival rate of the patients with gastric carcinoma. ${ }^{9} 10$ GOLPH3 is expressed at high levels in gastric carcinoma, but exists at low levels in normal tissues. However, there has been no report on the relationship of miR-126 with GOLPH3. The current study used gastric cancer BGC- 823 cells as the experimental object in the exploration of the role of miR-126 in the invasion and epithelial-mesenchymal transition (EMT) of gastric cancer cells, to provide a reference for the role of miR-126 in gastric cancer metastasis, and to provide new directions for targeted gene therapy for gastric cancer.

\section{Materials and Methods}

\section{Patients}

We analyzed the data of 45 patients who were diagnosed with gastric cancer at the First Affiliated Hospital of Guangzhou Medical University from August 2017 to March 2020. Those who had received radiotherapy and chemotherapy or who had other concomitant systemic tumors and chronic diseases were excluded. The ages ranged from 21-78 years, with a mean of $51.80 \pm 13.06$ years. The blood research control group consisted of 43 healthy medical examiners, aged 24 to 78 years, with a mean age of $51.42 \pm 14.31$ years.

\section{Cell culture and transfection}

The American Type Culture Collection (ATCC, USA) provided gastric cancer BGC-823 cells cultured in Dulbecco's modified eagle's medium (Sigma-Aldrich, St. Louis, MO, USA) with $1 \%$ penicillin/streptomycin and $10 \%$ fetal bovine serum (SigmaAldrich). Afterwards, the transfection of oligonucleotides (miR126 mimics, NC-mimics, miR-126 inhibitor, NC-inhibitor and siGOPLH3, respectively) and Lipofectamine 2000 (Invitrogen, Carlsbad, CA, USA) was used according to the manufacturer's instructions.

\section{Immunohistochemistry}

Ten percent formaldehyde was used to fix gastric cancer and normal gastric tissue adjacent to the cancer, which were embedded into wax blocks and sliced. The sections were dewaxed and rehydrated, then the antigen was retrieved.

The slices were put into the solution containing $3 \%$ hydrogen peroxide and incubated in dark at room temperature for $30 \mathrm{~min}$. Then washed in PBS solution for 3 times on a decolorizing shaker for $5 \mathrm{~min}$ each time. The sections were incubated at $4{ }^{\circ} \mathrm{C}$ overnight with a primary antibody recognizing GOLPH3 protein (Abcam, Cambridge, UK; ab236296, 1:100) followed by an incubation with a secondary antibody (Abcam; ab205718, 1:2000) for $30 \mathrm{~min}$ at room temperature. After the slices were slightly dried, the DAB solution was dripped into the circle. Under the microscope, the color development time was controlled (generally within $2 \mathrm{~min}$ ), and the positive color was brown yellow. Finally, the sections were counterstained with hematoxylin and dehydrated. Positively stained cells were those that appeared brownish yellow or yellowish brown under the microscope. Immunohistochemical results were quantified by Image Pro Process. Five fields were randomly selected under $\times 400$ magnification, 200 cells/field were randomly counted, and after calculation, the average positive rate was calculated and combined with the degree of staining and the average positive rate for semi-quantitative analysis. Quantitative analysis was performed with Image Pro plus software (Media Cybernetics, Silver Spring, MD, USA). Both positive (tissue slides of the stomach) and negative (incubating slides only with secondary antibody) controls were included in immunohistochemistry.

\section{MTT assay}

Cell viability was analyzed with the conventional MTT assay. The cells had now grown at $2-4 \times 10^{4}$ cells per well in 96-well microplates. MTT solution (Sigma-Aldrich) was then added to the medium to a final concentration of $0.5 \mathrm{mg} / \mathrm{mL}$ and incubated at $37^{\circ} \mathrm{C}$ for $4 \mathrm{~h}$. Measuring absorbance at $450 \mathrm{~nm}$ was calculated.

\section{Transwell cell detects invasion changes}

The steps of the invasion experiment were as follows: all groups were diluted with a serum-free culture medium; the density $\left(5 \times 10^{5}\right.$ cells $\left./ \mathrm{mL}\right)$ was added to the upper chamber; $200 \mu \mathrm{L}$ per well was added; then $500 \mu \mathrm{L}$ of the culture medium containing serum was pipetted into the lower chamber. After $24 \mathrm{~h}$ of culture, the chamber was removed; cells without membrane were wiped via a cotton swab, washed with PBS, fixed with paraformaldehyde, and stained with crystal violet.

\section{Target gene prediction and identification}

Using the target gene prediction software (Targetscan), miR126 was input and its target gene was detected, which was found to be probably GOPLH3. The luciferase report system was used to identify the relationship with this target gene. First, the pGL3GOPLH3-WT wild-type luciferase reporter vector containing the GOPLH3 3'UTR binding site was constructed, then the mutant pGL3- GOPLH3-MUT mutant luciferin without the GOPLH3 3'UTR binding site Enzyme reporter vector was constructed; WT, and MUT were co-transfected with HEK-293 cells with miR-126 mimics and mimics control, respectively. After the transfection for $24 \mathrm{~h}$, the change of luciferase activity was analyzed using the Luciferase Reporter Assay System.

\section{Quantitative RT-PCR}

The total RNA was extracted by the Trizol reagent (Invitrogen). Then, the SuperScript RT kit from Invitrogen was used to reverse transcribe $2 \mu \mathrm{g}$ of total RNA from the samples to cDNA. ABI PRISM7900 Sequence Detection System (Applied Biosystems, Waltham, MA, USA) with PowerUp ${ }^{\mathrm{TM}}$ SYBR ${ }^{\circledR}$ Green Master Mix (Thermo Fisher Scientific, Waltham, MA, USA) was used to perform quantitative RT-PCR. The $2^{-\Delta \Delta C t}$ method was used for the analysis of the relative expression levels of mRNA and miRNA, with GAPDH serving as an internal control. 


\section{Western blotting}

RIAP protein cleavage reagent was added in all groups; the protein supernatant was collected by ultrasonic disruption, and the protein concentration was determined by the BCA method. The mass concentration of the SDS-PAGE gel was $100 \mathrm{~g} / \mathrm{L}$. Protein samples were collected, mixed with Loading and Buffer, and placed in a boiling water bath for $5 \mathrm{~min}$. At a constant voltage of $90 \mathrm{~V}$ electrophoresis, bromophenol blue entered the bottom edge of the glass plate, and the gel was removed and placed in the transfer buffer. The PVDF membrane was cut to a suitable size, $300 \mathrm{~mA}$ was transferred with current, with a transfer time of $45 \mathrm{~min}$. The PVDF membrane was placed in $50 \mathrm{~g} / \mathrm{L}$ skim milk powder for $1 \mathrm{~h}$, and then incubated with primary antibody and secondary antibody reaction solutions. Antibodies were diluted with blocking solution; the dilution concentration of secondary antibody was 1:4000, and the dilution concentration of primary antibodies of E-cadherin, vimentin and $\mathrm{N}$-cadherin was 1:800. After color development with ECL, the Image $\mathrm{J}$ software was used to analyze the gray value of the target band E-cadherin, vimentin, $\mathrm{N}$-cadherin and the internal reference GAPDH gray value.

\section{Statistical analysis}

The experiment was repeated three times and the average value was taken. SPSS 21.0 statistical software was used for analysis. Measurement data were presented by mean $\pm \mathrm{SD}(\mathrm{x} \pm \mathrm{s})$. The compar- ison between the two groups was done by the $t$-test. The comparison of multiple groups was done via single factor analysis of variance. The $\mathrm{p}$ value $<0.05$, the difference was statistically significant.

\section{Results}

\section{Expression of miR-126 and GOLPH3 in gastric cancer cells and tissues}

GOLPH3 is mainly expressed in the cytoplasm, with a few in the cell membrane, as shown in Figure 1A. In comparison with normal gastric tissues, GOLPH3 was found to be highly expressed in gastric cancer. The qRT-PCR results also showed that GOLPH3 mRNA was highly expressed in gastric cancer BGC-823 cells, while miR-126 was significantly lowered (Figure 1B). We further used the Western blot to detect the GOLPH3 protein expression levels in gastric cancer cell BGC-823 and normal gastric cell RGM-1. The results showed that GOLPH3 protein was significantly overexpressed in BGC-823 cells (Figure 1C).

\section{Overexpression of miR-126 can inhibit the prolifera- tion, invasion and EMT of gastric cancer cell BGC-823}

After the transfection of miR-126 mimics in gastric cancer BGC-823 cells, the level of miR-126 in the cells increased (Figure
A

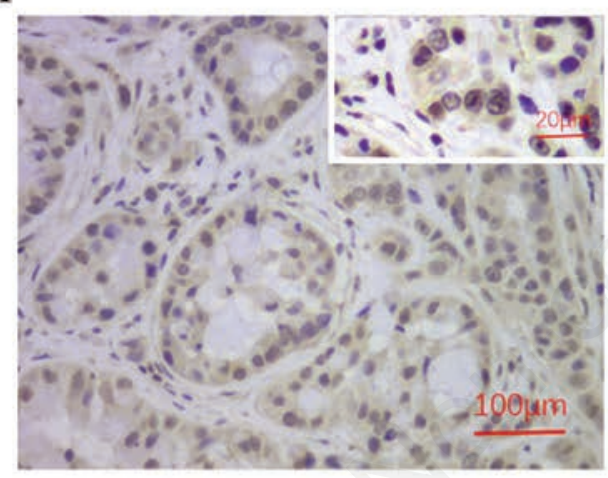

$\mathrm{B}$

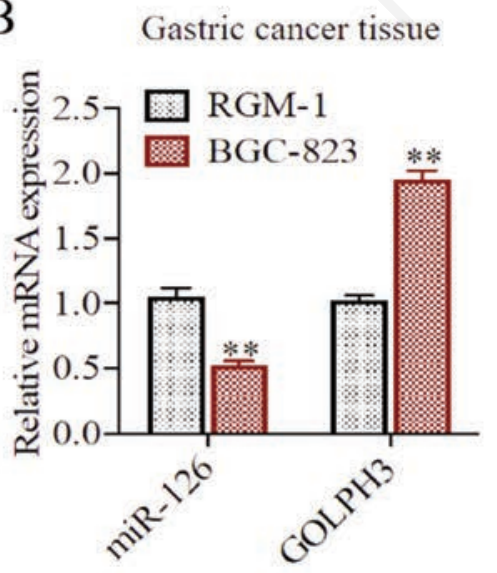

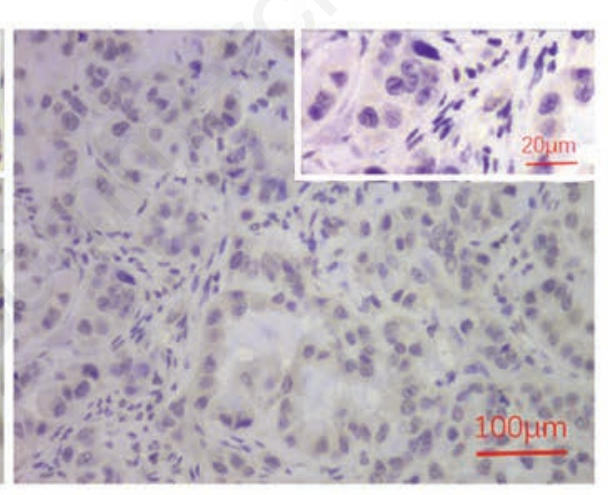

Normal tissue





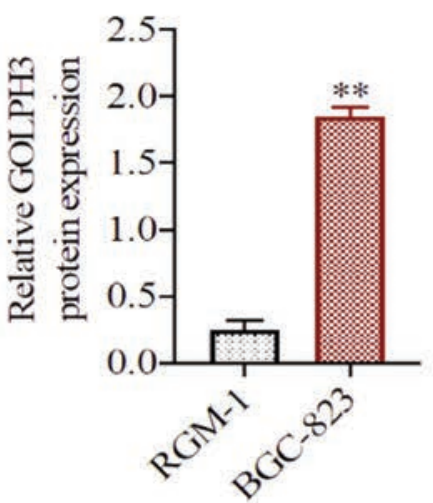

Figure 1. Expression of miR-126 and GOLPH3 in gastric cancer cells and tissues. A) Immunohistochemistry evaluation of the GOLPH3 expression. B) qRT-PCR evaluation of miR-126 and GOLPH3 mRNA expression. C) Western blot evaluation of the GOLPH3 protein expression. ${ }^{* *} \mathrm{p}<0.01$ vs RGM-1 group. 
2A), indicating that miR-126 mimics increased the expression level of miR-126 in gastric cancer BGC-823 cells. In addition, cell viability and invasion number were significantly reduced after the transfection of miR-126 mimics in gastric cancer BGC-823 cells (Figure 2 B,C), along with a decrease in the expression levels of vimentin and $\mathrm{N}$-cadherin protein in cells, while E-cadherin protein expression level was increased (Figure 2D). The above results indicate that miR-126 mimics inhibits gastric cancer BGC-823 cell proliferation, invasion and EMT.

\section{miR-126 targeted the regulation of GOLPH3 expression}

The online target gene prediction software predicted that miR-
A

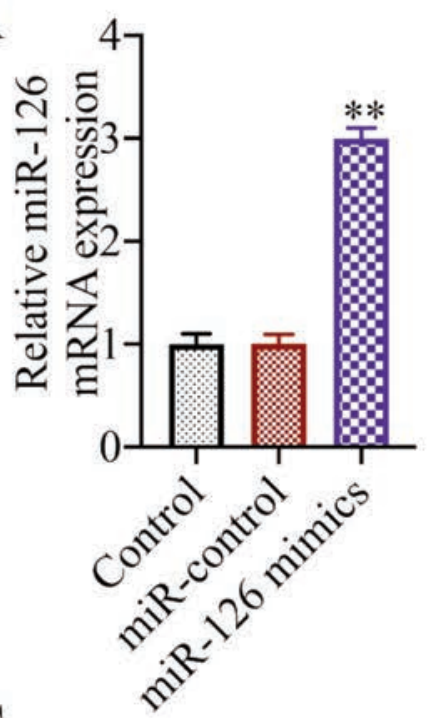

$\mathrm{B}$

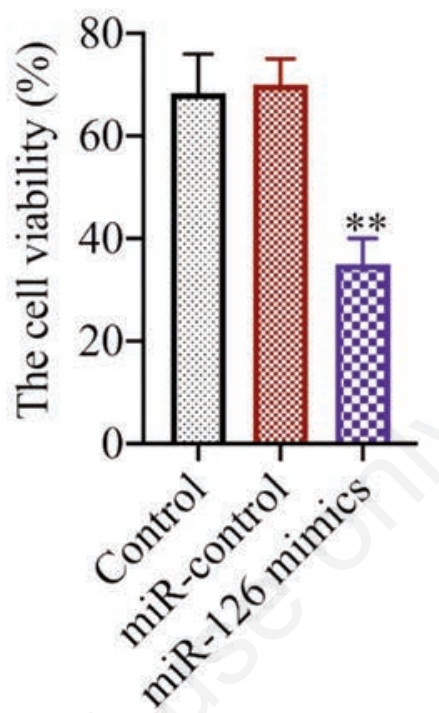

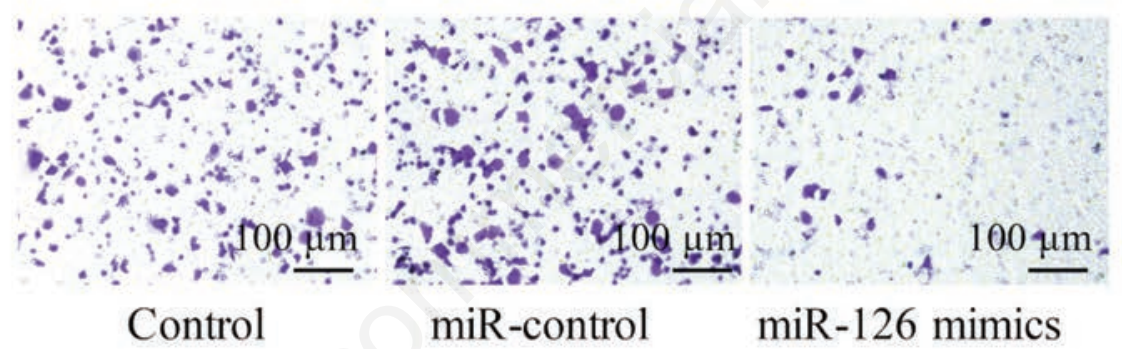

D

Control

miR-control
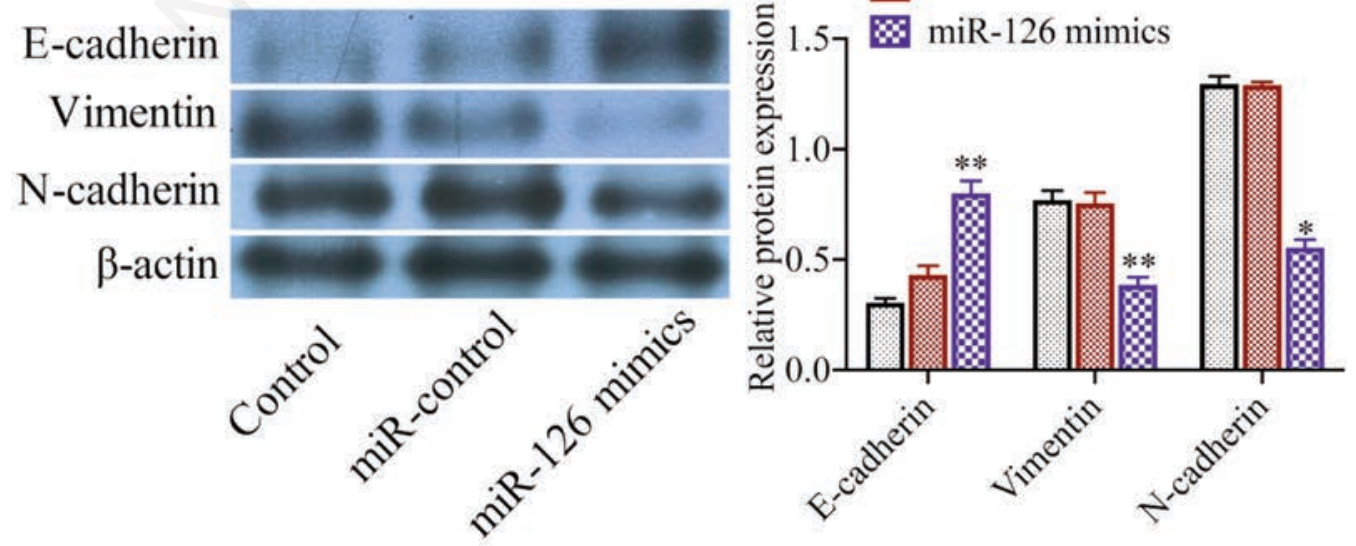

Figure 2. Overexpression of miR-126 can inhibit the proliferation, invasion and EMT of gastric cancer cell BGC-823. A) qRT-PCR evaluation of miR-126 mRNA expression. B) MTT assay evaluation of the cell viability. C) Transwell evaluation of the invasion of cells; blue represents the number of penetrating cells: the larger the number of penetrating cells, the stronger the invasive ability of cells. $\mathrm{D}$ ) Western blot evaluation of the E-cadherin, vimentin and $\mathrm{N}$-cadherin protein expressions. ${ }^{*} \mathrm{p}<0.05,{ }^{* *} \mathrm{p}<0.01 v s$ control group. 
A GOLPH3_WT 5'- ACGTGTACAGGATTCTGCTGGTACGAG -3'

hsa-miR-126-3p

\author{
3'-GCGUAAUAAUGAGUGCCAUGCU-5'
}

GOLPH3_MUT 5'- ACGTGTACAGGATTCTGCDTGAGTAGG -3'

B

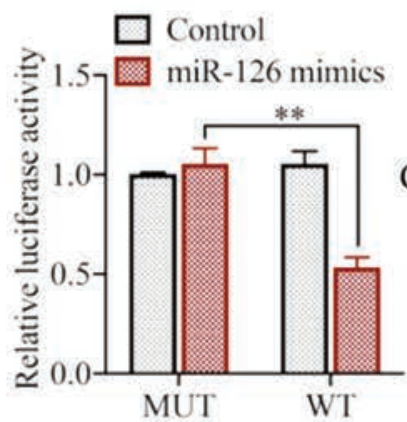

$\mathrm{C}$

GOLPH3

$\beta$-actin
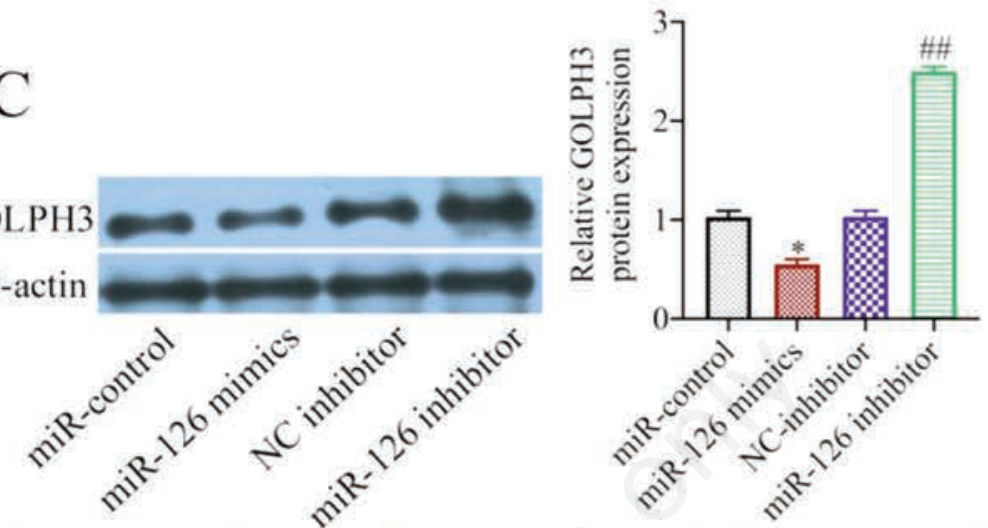

D

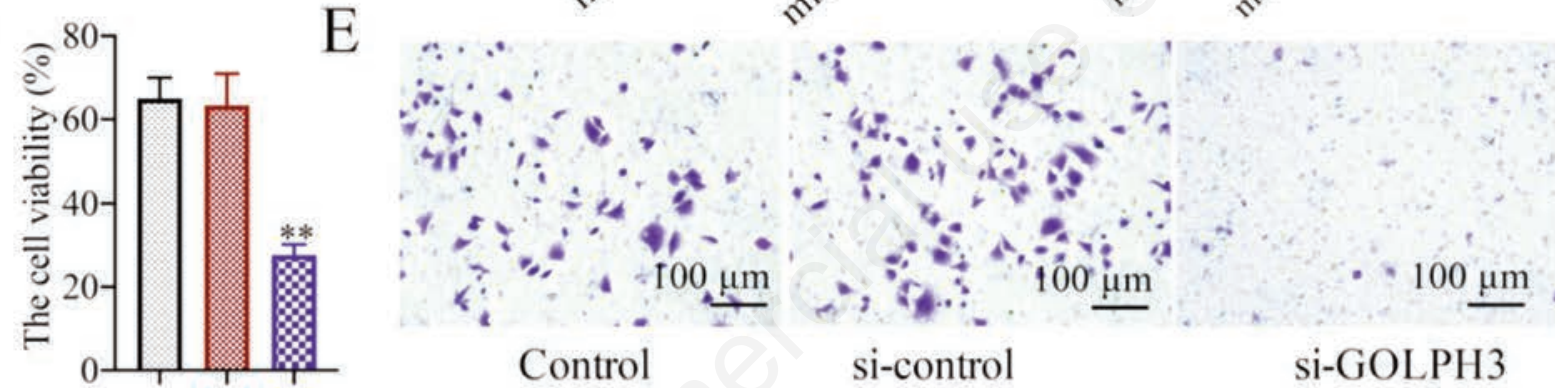

F
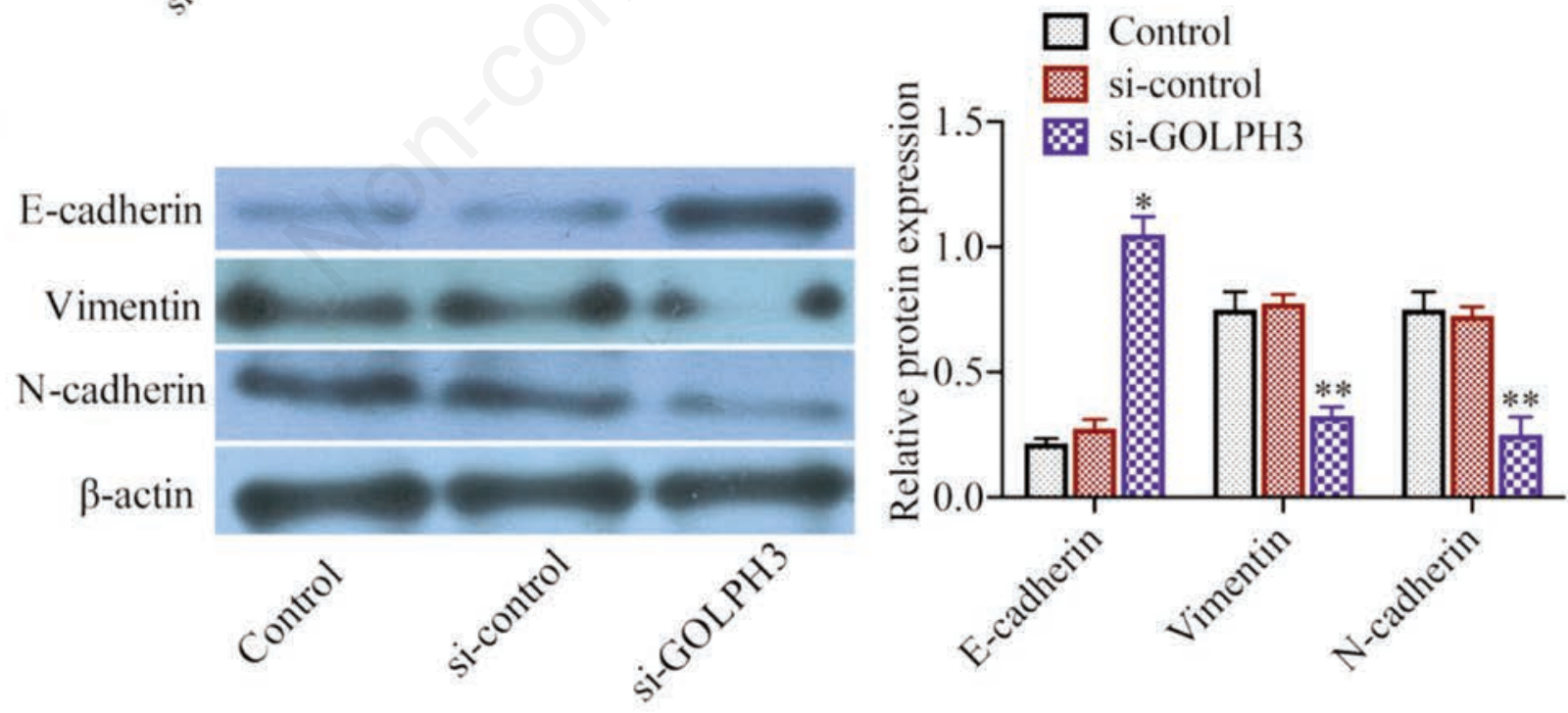

Figure 3. miR-126 targeted the regulation of GOLPH3 expression. A) TargetScan predicts miR-126 and GOLPH3 binding sites. B) Luciferase activity analysis of the targeted relationship of miR-126 and GOLPH3 in HEK-293T cells. C) The GOLPH3 protein expression was evaluated by Western blot; blue represents the number of penetrating cells: the larger the number of penetrating cells, the stronger the invasive ability of cells. D) The cell viability evaluated by MTT assay. E) The invasion of cells was evaluated by Transwell. F) The E-cadherin, vimentin and $\mathrm{N}$-cadherin protein expressions evaluated by Western blot. ${ }^{* *} \mathrm{p}<0.01$ vs control group, \#\#p<0.01 vs $\mathrm{NC}$ inhibitor. 
126 and GOLPH3 had a binding site at the 3'UTR end (Figure $3 \mathrm{~A})$. WT and miR-126 mimics were co-transfected into gastric cancer BGC-823 cells with a resulting decrease in the luciferase activity (Figure 3B), and a decrease in the expression level of GOLPH3 protein in cells transfected with miR-126 mimics (Figure $3 C)$. Moreover, after the transfection of si-GOLPH3 in gastric cancer BGC-823 cells, there was a significant increase in the cell viability and invasion number (Figure $3 \mathrm{D}, \mathrm{E}$ ), the expression levels of vimentin and $\mathrm{N}$-cadherin protein in cells also increased, while the E-cadherin expression was reduced (Figure 3E).
Overexpression of GOLPH3 can partially reverse the effect of miR-126 on proliferation, invasion and EMT of gastric cancer cell

In order to further study the effects of GOLPH3 and miR-126 on the proliferation, invasion and EMT of gastric cancer cell BGC823 , we co-transfected BGC-823 cells with overexpressed miR126 and overexpressed GOLPH3 plasmids to detect cell proliferation, invasion and EMT. Compared with cells co-transfected with pcDNA 3.0 and miR-126 mimics, pcDNA co-transfected 3.0GOLPH3 and miR-126 mimics into gastric cancer BGC-823 cells,
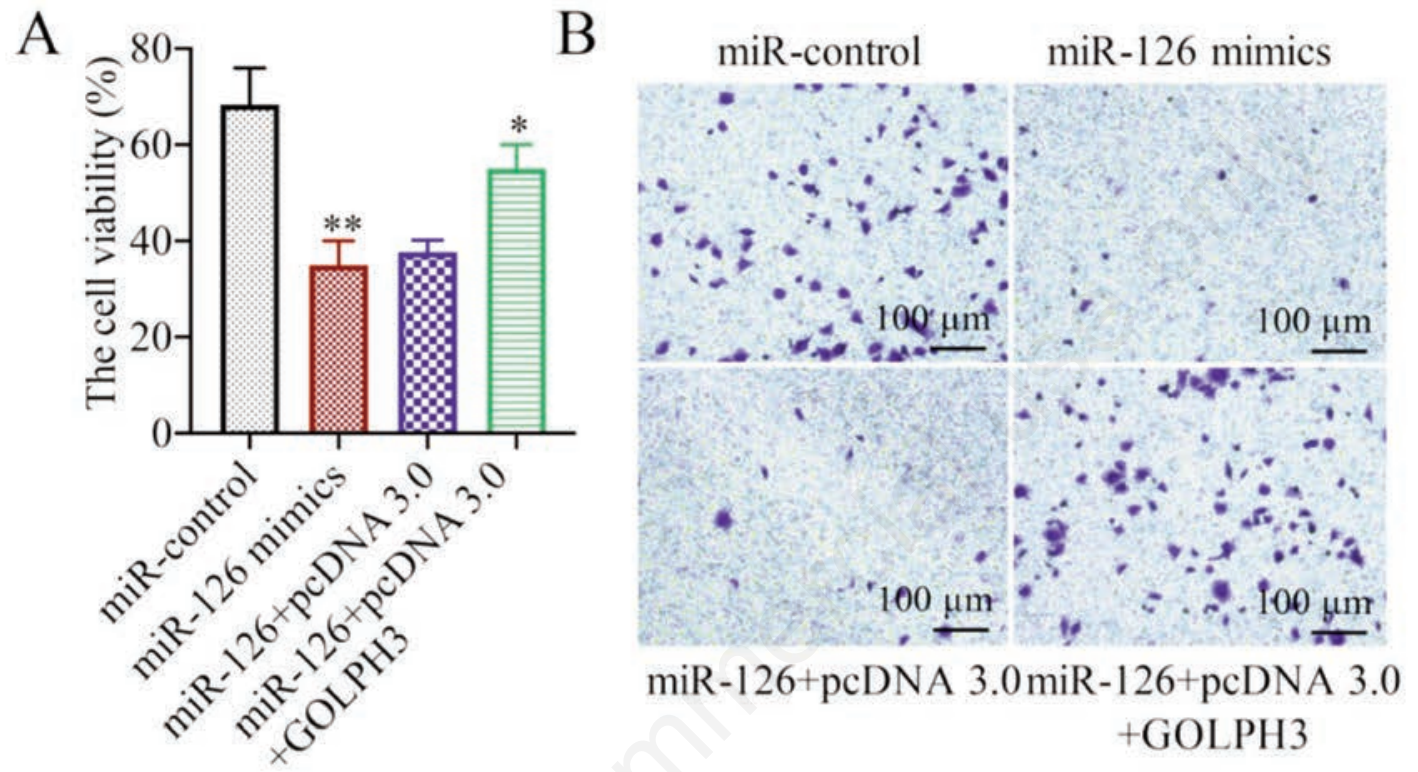

C
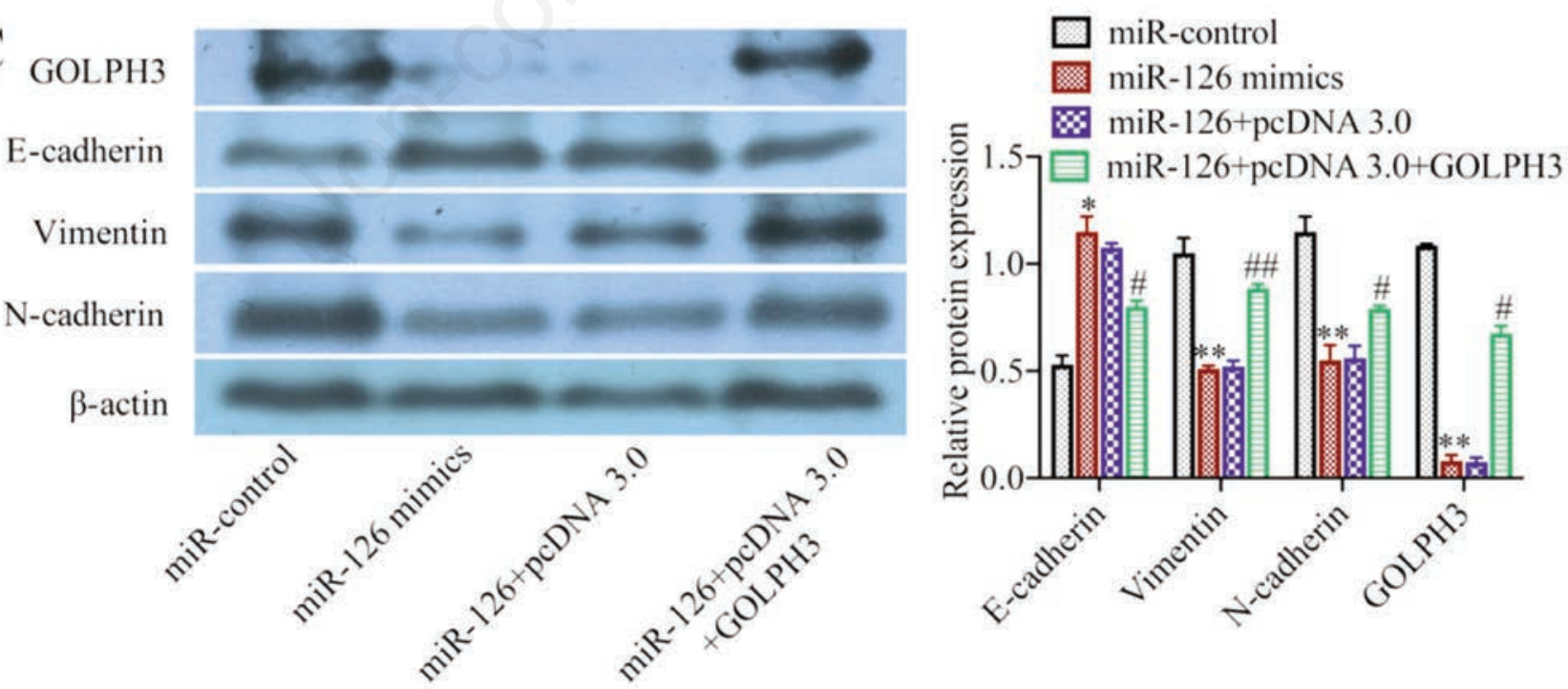

Figure 4. Overexpression of GOLPH3 can partially reverse the effect of miR-126 on proliferation, invasion, migration and EMT of gastric cancer cell. A) The cell viability was evaluated by MTT assay. B) The invasion of cells was evaluated by Transwell. D) The E-cadherin, vimentin and $\mathrm{N}$-cadherin protein expressions evaluated by Western blot. ${ }^{*} \mathrm{p}<\mathbf{0 . 0 5},{ }^{* *} \mathrm{p}<\mathbf{0 . 0 1} v \boldsymbol{v}$ miR-control group, \#p<0.05, $\#$ \# $<0.01$ vs miR-126 + pcDNA 3.0 group. 
with a resulting increase in the cell viability and invasion number (Figure 4 A,B), E-cadherin protein levels decreased, while Vimentin and N-cadherin protein levels increased (Figure 4C).

\section{Discussion}

miRNA molecules, which are important biological regulators, are expressed in animals and plants, and participate in the processes of cell differentiation, growth, and apoptosis. ${ }^{11}$ Recent studies have linked miRNA to the occurrence of diseases such as diabetes and atherosclerosis. ${ }^{12,13}$ In recent years, miRNAs have regulated tumorigenesis and metastasis, which has risen a widespread concern. miRNAs are abnormally expressed in tumors and play a role either as oncogenes or tumor suppressor genes in tumor progression. ${ }^{14}$ Some studies have compared nine gastric cancer cell lines detected by the miRNAs microarray with normal gastric tissues, and miR-126 was significantly downregulated. ${ }^{15}$ Yue et al. also found that miR-126 expression in gastric cancer tissues decreased significantly, and in vitro gastric cancer cells could inhibit the growth of SGC7901 cells by inducing the G0/G1 phase to block the cell cycle. ${ }^{16}$ Current research on miR-126 in gastric cancer showed that it was lowly expressed in gastric cancer tissues and gastric cancer cells, which could result in a blockage of the gastric cancer cell cycle and an induction of gastric cancer cell apoptosis, and may consequently play an inhibitory role in the progress of gastric cancer. Moreover, our experiments have shown that gastric cancer cell proliferation and invasion capacity are decreased after upregulating miR-126 expression, indicating that miR-126 plays an inhibitory role in the malignant phenotype of gastric cancer cells, and it can equally inhibit the metastatic potential of gastric cancer cells.

Tumor cell invasion and cell biological characteristics are closely related to tumor metastasis. As researchers continue to study the mechanism of tumor metastasis, the EMT of tumor cells occurs before tumor metastasis, and the potential of tumor cell metastasis of EMT is greater. ${ }^{17,18}$ EMT is the gradual disappearance of epithelial cells. In the process of gradually showing the characteristics of interstitial cells E-cadherin acts as an epithelial cell marker; vimentin and $\mathrm{N}$-cadherin are interstitial cell markers, and changes in their expressions are the indicators of cellular EMT levels. ${ }^{18,19}$ The process of EMT is very complex and usually influenced by genes and the external environment, such as helicobacter might induce EMT. ${ }^{20}$ In our experiment, it was found that E-cadherin protein levels were increased and Vimentin and N-cadherin protein levels were decreased after upregulation of miR-126, indicating that the upregulation of miR-126 can reduce the EMT levels of gastric cancer cells, and miR-126 has the effect of reducing the metastatic potential of gastric cancer cells.

Research on the regulatory mechanism of miRNA found that it exerts a variety of biological functions by affecting the expression of downstream target genes and may contain multiple target genes at the same time. miR-126 may be closely related to the inhibition of gastric cancer progression. ${ }^{21}$ In the study of esophageal squamous cell carcinoma, Li et al. found that miR-126 inhibits the proliferation and invasion of tumor cells by regulating GOLPH3. It is speculated that GOLPH3 may be the target gene of miR-126. ${ }^{16,22}$ Studies have found that GOLPH3 plays an important role in Golgi vesicle transport and Golgi structure. ${ }^{22}$ We found that miR-126 negatively regulates GOLPH3 and upregulates and targets GOLPH3 protein expression. Our experiments also confirmed that GOLPH3 could reverse the upregulation of miR-126 on gastric cancer cell proliferation, invasion, and EMT. This indicates that miR-126 can inhibit gastric cancer cell proliferation, invasion and EMT by targeting GOLPH3 expression. There are also limitations of this study. For example, additional in vivo experiments are needed to verify the in vitro results.

In conclusion, miR-126 plays an inhibitory role in gastric cancer cell proliferation, invasion and migration, EMT, and gastric cancer metastasis. Its mechanism of action is related to targeted downregulation of GOLPH3 expression. The regulatory role in the progression and metastasis of gastric cancer provides a reference for laying a foundation for studying the miR-126 network regulatory mechanism. However, this assumption is based on the results of our study and these findings probably requires further investigations as well.

\section{References}

1. Bray FI, Ferlay J, Soerjomataram I, Siegel RL, Torre LA, Jemal A. Global cancer statistics 2018: GLOBOCAN estimates of incidence and mortality worldwide for 36 cancers in 185 countries. CA Cancer J Clin 2018;68:394-424.

2. Seyfried TN, Huysentruyt LC. On the origin of cancer metastasis. Crit Rev Oncog 2013;18:43-73.

3. Grenda A, Krawczyk P. New dancing couple: PD-L1 and microRNA. Scand J Immunol 2017;86:130-4.

4. Luo M, Li L. Clinical utility of miniprobe endoscopic ultrasonography for prediction of invasion depth of early gastric cancer: A meta-analysis of diagnostic test from PRISMA guideline. Medicine (Baltimore) 2019;98:e14430.

5. Narita Y, Muro K. Challenges in molecular targeted therapy for gastric cancer: considerations for efficacy and safety. Expert Opin Drug Saf 2017;16:319-27.

6. Scott KL, Kabbarah O, Liang M, Ivanova E, Anagnostou V, $\mathrm{Wu} \mathrm{J}$, et al. GOLPH3 modulates mTOR signalling and rapamycin sensitivity in cancer. Nature 2009;459:1085-90.

7. Aleckovic M, Kang Y. Regulation of cancer metastasis by cellfree miRNAs. Biochim Biophys Acta 2015;1855:24-42.

8. Chen M, Peng W, Hu S, Deng J. miR-126/VCAM-1 regulation by naringin suppresses cell growth of human non-small cell lung cancer. Oncol Lett 2018;16:4754-60.

9. Wang P, Li Z, Liu H, Zhou D, Fu A, Zhang E. MicroRNA-126 increases chemosensitivity in drug-resistant gastric cancer cells by targeting EZH2. Biochem Bioph R Co 2016;479:91-6.

10. Yang Z, Wang R, Zhang T, Dong X. MicroRNA-126 regulates migration and invasion of gastric cancer by targeting CADM1. Int J Clin Exp Pathol 2015;8:8869-80.

11. Hsu W, Li W, Lee Y, Huang A, Chang L, Lin H, et al. MicroRNA-145 suppresses cell migration and invasion in upper tract urothelial carcinoma by targeting ARF6. FASEB J 2020;34:5975-92.

12. Brennan EP, Wang B, Mcclelland A, Mohan M, Marai M, Beuscart $\mathrm{O}$, et al. Protective effect of let-7 miRNA family in regulating inflammation in diabetes-associated atherosclerosis. Diabetes 2017;66:2266-77.

13. Lopes MB, De Freitas RCC, Hirata MH, Hirata RDC, De Rezende AA, Silbiger VN, et al. mRNA-miRNA integrative analysis of diabetes-induced cardiomyopathy in rats. Front Biosci (Schol Ed) 2017;9:194-229.

14. Wang M, Lv G, Jiang C, Xie S, Wang G. miR-302a inhibits human HepG2 and SMMC-7721 cells proliferation and promotes apoptosis by targeting MAP3K2 and PBX3. Sci Rep 2019;9:2032.

15. Yue S, Shi H, Han J, Zhang T, Zhu W, Zhang D. Prognostic value of microRNA-126 and CRK expression in gastric cancer. Onco Targets Ther 2016;9:6127-35.

16. Li H, Meng F, Ma J, Yu Y, Hua X, Qin J, et al. Insulin receptor substrate-1 and Golgi phosphoprotein 3 are downstream tar- 
gets of miR-126 in esophageal squamous cell carcinoma. Oncol Rep 2014;32:1225-33.

17. Krebs AM, Mitschke J, Losada ML, Schmalhofer O, Boerries $\mathrm{M}$, Busch H, et al. The EMT-activator Zeb1 is a key factor for cell plasticity and promotes metastasis in pancreatic cancer. Nat Cell Biol 2017;19:518-29.

18. Passacantilli I, Panzeri V, Bielli P, Farini D, Pilozzi E, Fave $\mathrm{GD}$, et al. Alternative polyadenylation of ZEB1 promotes its translation during genotoxic stress in pancreatic cancer cells. Cell Death Dis 2017;8:e3168.

19. Sun L, Ke J, He Z, Chen Z, Huang Q, Ai W, et al. HES1 Promotes colorectal cancer cell resistance to 5-fu by inducing of EMT and
ABC transporter proteins. J Cancer 2017;8:2802-8.

20. Baj J, Korona-Głowniak I, Forma A, Maani A, Sitarz E, Rahnama-Hezavah M, et al. Mechanisms of the epithelial-mesenchymal transition and tumor microenvironment in Helicobacter pylori-induced gastric cancer. Cells 2020;9:1055.

21. Holubekova V, Mendelova A, Jasek K, Mersakova S, Zubor P, Lasabova Z. Epigenetic regulation by DNA methylation and miRNA molecules in cancer. Future Oncol 2017;13:2217-22.

22. Sechi S, Frappaolo A, Belloni G, Colotti G, Giansanti MG. The multiple cellular functions of the oncoprotein Golgi phosphoprotein 3. Oncotarget 2015;6:3493-506. 